\title{
RAGE and HMGB1 Expression in Orbital Tissue Microenvironment in Graves' Ophthalmopathy
}

\author{
Dominika Lacheta, ${ }^{1}$ Krzysztof B. Poślednik, ${ }^{2}$ Katarzyna Czerwaty, ${ }^{2}$ Nils Ludwig, ${ }^{3}$ \\ Marta Molińska-Glura, ${ }^{4}$ Ireneusz Kantor, ${ }^{2}$ Anna Jabłońska-Pawlak (D), ${ }^{5}$ Piotr Miśkiewicz, ${ }^{6}$ \\ Alicja Głuszko, ${ }^{1}$ Zygmunt Stopa $\mathbb{D}^{D}{ }^{7}$ Jacek Brzost, ${ }^{8}$ and Mirosław J. Szczepański $\mathbb{D}^{1,2}$ \\ ${ }^{1}$ Department of Biochemistry, Medical University of Warsaw, 02-097 Warsaw, Poland \\ ${ }^{2}$ Department of Otolaryngology, Medical Centre for Postgraduate Education, 03-242 Warsaw, Poland \\ ${ }^{3}$ Department of Oral and Maxillofacial Surgery, University Hospital Regensburg, 93053 Regensburg, Germany \\ ${ }^{4}$ Department of Forest Technology of Poznan University of Life Sciences, 60-624 Poznan, Poland \\ ${ }^{5}$ Department of Ophthalmology, Medical University of Warsaw, 02-097 Warsaw, Poland \\ ${ }^{6}$ Department of Internal Medicine and Endocrinology, Medical University of Warsaw, 02-097 Warsaw, Poland \\ ${ }^{7}$ Department of Maxillofacial Surgery, Medical University of Warsaw, 02-097 Warsaw, Poland \\ ${ }^{8}$ Department of Otolaryngology, The Children's Memorial Health Institute, 04-730 Warsaw, Poland \\ Correspondence should be addressed to Mirosław J. Szczepański; mszczepanski@wum.edu.pl
}

Received 29 September 2020; Revised 31 December 2020; Accepted 18 February 2021; Published 12 March 2021

Academic Editor: Liliana Paris

Copyright (c) 2021 Dominika Łacheta et al. This is an open access article distributed under the Creative Commons Attribution License, which permits unrestricted use, distribution, and reproduction in any medium, provided the original work is properly cited.

\begin{abstract}
Graves' ophthalmopathy (GO) is a chronic autoimmune inflammatory disorder involving orbital tissues. A receptor for advanced glycation end products (RAGE) and its ligand high mobility group box 1 (HMGB1) protein trigger inflammation and cell proliferation and are involved in the pathogenesis of various chronic inflammatory diseases. This study was aimed to evaluate RAGE and HMGB1 expression in GO to determine its potential clinical significance. To the best of our knowledge, this is the first study showing RAGE and HMGB1 expression in orbital tissue using immunohistochemistry. Sections of orbital adipose tissue obtained from patients diagnosed with GO (23 patients; 36 orbits) and normal controls (NC) (15 patients; 15 orbits) were analyzed by immunohistochemistry for RAGE and HMGB1 expression. Expression profiles were then correlated with clinical data of the study group. RAGE and HMGB1 expression were elevated in GO patients in comparison with NC ( $p=0.001$ and $p=0.02$, respectively). We observed a correlation between RAGE expression and occurrence of dysthyroid optic neuropathy $(\mathrm{DON})(p=0.05)$ and levels of TSH Receptor Antibodies (TRAb) $(p=0.01)$. Overexpression of RAGE and HMGB1 might be associated with GO pathogenesis. In addition, RAGE and HMGB1 proteins may be considered as promising therapeutic targets, but this requires further research.
\end{abstract}

\section{Introduction}

Graves' disease (GD) represents an autoimmune process in which circulating autoantibodies directed against thyrotropin receptor (TSHR)-TRAb (TSHR antibodies) - activate the thyroid gland, causing hyperthyroidism [1]. One of the extrathyroidal symptoms of GD is Graves' ophthalmopathy (GO), defined as a chronic autoimmune inflammatory disor- der involving orbital tissues [2]. Although patients with GO are mostly hyperthyroid, they can also be euthyroid or hypothyroid. Moreover, GO may be reported in Hashimoto's thyroiditis [3]. Cytokine production, inflammatory infiltration, and orbital fibroblast activity result in expansion and remodeling of extraocular tissues-mainly orbital adipose tissue and fibrous tissue of extraocular muscles. Edematousinfiltrative changes involving orbital soft tissues are observed 
in $25-50 \%$ of patients with GD [4]. Clinical manifestations of GO include lid retraction, double vision, soft tissue swelling, and erythema of the conjunctival and periorbital tissues. Increased intraocular pressure within the inflexible bony orbital walls can contribute to the development of proptosis and optic nerve compression, including dysthyroid optic neuropathy (DON). According to the European Group on Graves' Orbitopathy (EUGOGO), severity of GO is rated as mild, moderate-to-severe, and sight threatening (including DON and/or severe keratitis) [5].

Multiligand receptor for advanced glycation end products (RAGE) is suggested to initiate and amplify immune and inflammatory responses [6]. Increased levels of RAGE ligands in chronic disorders indicate that RAGE is involved in the pathogenesis of various inflammatory diseases [7]. Cellular stress causes the generation of RAGE ligands such as high mobility group box 1 (HMGB1) protein, S100 proteins, and nucleic acids, while prolonged hyperglycemia and inflammation induce the release of the ligands AGE and amyloid [8]. HMGB1 is one of the most significant members of the DAMP (damage-associated molecular patterns) family. DAMPs involve molecules released by dying or necrotic cells and can induce inflammation, cell proliferation, and migration [9]. HMGB1-RAGE interaction affects inflammation via the activation of proinflammatory transcription factor $\mathrm{NF}-\kappa \mathrm{B}$ (nuclear factor kappa B) which furthermore regulates RAGE [10].

In this study, we test the hypothesis that RAGE and HMGB1 are overexpressed in orbit tissue in GO and that expression patterns correlate with disease severity. To the best of our knowledge, this is the first study showing RAGE and HMGB1 expression in orbital tissue by immunohistochemistry.

\section{Materials and Methods}

2.1. Patients and Tissue Collection. Archival tissue paraffin blocks were used for the studies. All orbital adipose tissue samples used in the study were collected during surgery for routine histopathological examination between 2016 and 2020. Tissues were obtained from 23 patients ( 18 females, 5 males, 36 orbits) diagnosed with GO who underwent transnasal endoscopic orbital decompression surgery in the Otolaryngology Department, Centre of Postgraduate Medical Education in Warsaw, Poland, or were derived from a tissue bank at the Department of Internal Medicine and Endocrinology, Medical University of Warsaw, Poland, from previous studies. Inclusion criteria for the study were in accordance with the EUGOGO guidelines: (1) moderate-tosevere GO or (2) sight-threatening GO. Patients with any of the following conditions were excluded from the study: (1) age under 18 years, (2) a history of eye surgery, (3) a previous history of metabolic diseases or other diseases that may affect orbital connective tissues, except for thyroid disease, and (4) pregnancy or lactation. As controls, orbital adipose tissue samples were obtained from 15 patients (15 orbits) operated on the orbit due to trauma at the Department of Maxillofacial Surgery, Medical University of Warsaw, Poland. The study was approved by Local Ethics
Committees at the Medical University of Warsaw in Poland (\#AKBE/86/2018 to P.M.; KB/126/2016 to M.J.S) and at the Centre of Postgraduate Medical Education (\#16/PB/2018 to I.K.). Tissues were fixed in $10 \%$ formaldehyde and embedded in paraffin. Sections were stained with hematoxylineosin $(\mathrm{H}+\mathrm{E})$ and evaluated by light microscopy before performing immunohistochemistry.

2.2. Clinical Characteristics of Graves' Ophthalmopathy. Ocular involvement, including GO severity and activity, was defined as absent, mild, moderate-to-severe, and sightthreatening-active or inactive, according to criteria reported in EUGOGO recommendations [5, 11]. Patients were classified as "active" or "inactive" based on a Clinical Activity Score (CAS). Dysthyroid optic neuropathy (DON) was defined by visual dysfunction secondary to GO when other causes for visual impairment had been excluded. The diagnosis of DON was based on at least two criteria from the following: (1) reduced visual acuity $(<1.0)$, (2) relative afferent pupillary defect, (3) reduced color vision (more than two errors in Ishihara plates), (4) optic disc swelling in the affected eye, and (5) magnetic resonance imaging of orbit showing apical crowding or optic nerve stretching. Corneal involvement (keratitis) was defined as absent or punctuate keratopathy/ulcer. Best-corrected visual acuity (BCVA) was examined using Snellen charts and expressed as a decimal fraction. Exophthalmos was measured by a Hertel exophthalmometer. The intraocular pressure was measured in the primary position using an applanation tonometer. All the ophthalmology examinations for all patients were carried out by the same ophthalmologist. Clinicopathological characteristics of Graves' ophthalmopathy (GO) patients are presented in Table 1.

2.3. Immunohistochemistry. Paraffin sections of GO patients and NC patients were immunostained using NovoLink Polymer Detection Systems (Novocastra Laboratories, Newcastle, UK) and the following primary antibodies diluted $1: 100$ in Antibody Diluent (Dako): rabbit polyclonal anti-human HMGB1 (LS-C2691, LifeSpan BioSciences, Inc., Seattle, WA, USA) and mouse monoclonal anti-human RAGE (LSB6042, LifeSpan BioSciences, Inc., Seattle, WA, USA). Deparaffinated and rehydrated sections were stained according to the manufacturer's instructions, as previously described [12]. The activity of endogenous peroxidase was blocked by Peroxidase Block (NovoLink Polymer Detection System; Novocastra Laboratories). Sections were incubated with primary antibodies overnight and were subsequently incubated with Post Primary (rabbit anti-mouse IgG; NovoLink Polymer Detection System; Novocastra Laboratories) which detected mouse antibodies and with Polymer (NovoLink Polymer Detection System; Novocastra Laboratories) to recognize rabbit antibodies and detect Post Primary and finally with 3,3' -diaminobenzidine chromogen (NovoLink Polymer Detection System; Novocastra Laboratories). Nonspecific binding of the primary antibodies and the polymer was eliminated by application of Protein Block (NovoLink Polymer Detection System; Novocastra Laboratories) before adding the primary antibodies. Sections were counterstained with 
TABLE 1: Clinical characterization of the Graves' ophthalmopathy (GO) patients included in this study at the day of surgery.

\begin{tabular}{|c|c|c|}
\hline Characteristics & $\begin{array}{l}\text { Patients } \\
(n=23) \\
36 \text { orbits }\end{array}$ & $\begin{array}{c}\text { Normal controls } \\
\quad(n=15) \\
15 \text { orbits } \\
\end{array}$ \\
\hline \multicolumn{3}{|l|}{ Sex (number of patients) } \\
\hline Female & 18 & 4 \\
\hline Male & 5 & 11 \\
\hline \multicolumn{3}{|l|}{ Age (years) } \\
\hline Range & $35-73$ & $36-56$ \\
\hline Median & 65 & 46 \\
\hline \multicolumn{3}{|c|}{ Disease status (number of orbits) } \\
\hline Moderate-to-severe GO & 14 & \\
\hline \multicolumn{3}{|l|}{ Severe GO } \\
\hline With DON & 12 & \\
\hline With severe keratitis & 10 & \\
\hline $\begin{array}{l}\text { Graves' disease (number of } \\
\text { patients) }\end{array}$ & 23 & \\
\hline Median CAS & 2 & \\
\hline Thyroidectomy & 7 & \\
\hline Median proptosis (mm) & 23 & \\
\hline Median IOP (mmHg) & 16 & \\
\hline Median BCVA & 0.9 & \\
\hline Median fT4 (pmol/L) & 19.4 & \\
\hline Median TRAb (U/L) & 12.4 & \\
\hline Median aTPO (U/mL) & 89.3 & \\
\hline Median aTG $(\mathrm{U} / \mathrm{mL})$ & 144 & \\
\hline
\end{tabular}

Abbreviations: DON: dysthyroid optic neuropathy; CAS: clinical activity score; IOP: intraocular pressure; BCVA: best-corrected visual acuity; fT4: free thyroxine; TRAb: anti-TSHR antibodies; aTPO: antithyroid peroxidase; aTG: antithyroglobulin.

hematoxylin (Dako), dehydrated, coverslipped, and evaluated by light microscopy ZEISS Observer Z1 (Axiovision 4.8 software; illumination system LUMEN 200; PRIOR) in high power field (magnification $\times 400$ ). Results were scored by two independent investigators (M.J.S. and D.t.) as positive $(++)$, heterogeneous $(+)$, or negative $(-)$, when the number of stained cells in each section was $>75$ cells, between 25 and 75 cells, and $<25$ cells, respectively, as previously described [13].

2.4. Statistical Analysis. Statistical analysis was performed by professional statistician using the Statistica 13 software package. The Fisher exact test was used for the binary variable. When comparing continuous variable, without normal distribution (checked with a Kolmogorov-Smirnov test), differences between groups were measured using MannWhitney's $U$ test. The significance level was established at $p \leq 0.05$.

\section{Results}

3.1. RAGE and HMGB1 Expression in Tissue Sections. RAGE expression levels were elevated in GO tissues compared to those from NC ( $p=0.001$; Figure $1(\mathrm{a}))$. In GO tissues, RAGE was detected in the cytoplasm, and the expression was positive or heterogenous with staining intensities ranging from weak to strong. In contrast, RAGE expression was observed in only $25 \%$ of NC tissues and its staining intensity ranged from negative to weak (Figure 1(a)).

HMGB1 was detectable in the nuclei and cytoplasm in all tissues of GO patients and NC (Figure 1(b)). Intensity of HMGB1 staining was evaluated as moderate or strong (Figures 1(a) and 1(b)). Differences between GO and NC in terms of HMGB1 expression were statistically significant $(p=0.02$; Figure 1(a)).

3.2. RAGE Expression Correlates with Disease Severity. In the GO cohort, we observed differences in RAGE positivity depending on occurrence of DON and TRAb levels (Figure 2). GO patients with DON had stronger expression of RAGE (positivity “++") than tissues from patients without DON ( $p=0.05$ ) (Figure 2(a)). Moreover, RAGE staining correlated with TRAb levels in GO patients. Positivity “++" was characteristic for high levels of TRAb $(p=0.02$; Figure 2(b)).

The correlation of HMGB1 expression levels in the GO cohort with clinical data revealed no statistically significant differences $(p>0.05)$. However, we observed the tendency between increased levels of HMGB1 expression in patients with sight-threatening GO with DON (data not shown).

3.3. RAGE and HMGB1 Expression in Inflammatory Infiltrates. In GO tissues, we observed elevated numbers of inflammatory cells that expressed RAGE and HMGB1 proteins. Those polymorphonuclear and mononuclear cells were mainly localized in close proximity to the vessels (Figure 3).

\section{Discussion}

In our study, we demonstrated significant differences between GO and NC tissues for RAGE and HMGB1 expression. Enhanced expression of RAGE occurs in conditions of inflammatory mediators and ligands for RAGE accumulation [8]. Literature data indicate that the inflammatory process comprising orbital tissues underlies the pathogenesis of GO $[14,15]$. Immune cell infiltration as well as cytokine production activates orbital fibroblasts and GAG synthesis resulting in adipose tissue expansion. Yoon et al. have demonstrated that mRNA and protein levels of the proinflammatory cytokines TNF- $\alpha$ and IL- $1 \beta$ are increased in GO tissues compared to healthy controls [16]. Furthermore, our preliminary study has shown that a variety of proteins and cytokines involved in inflammation, autoimmunity, hypoxia, and fibrosis are expressed in GO orbital adipose tissue microenvironment, including TGF- $\beta$, TLR-4 (Toll-like receptor 4), HIF- $1 \alpha$ (hypoxia-inducible factor- $1 \alpha$ ), NF- $\kappa \mathrm{B}$ (nuclear factor kappa B), and IL-17 [14].

Current evidences point out that HMGB1 protein is involved in numerous chronic inflammatory and autoimmune diseases including rheumatoid arthritis, atherosclerosis, and systemic lupus erythematosus (SLE) [17, 18]. Released HMGB1 interacts with its cell-surface receptorRAGE, activating the main signaling pathways responsible for the pathogenesis of these diseases. HMGB1 is involved 

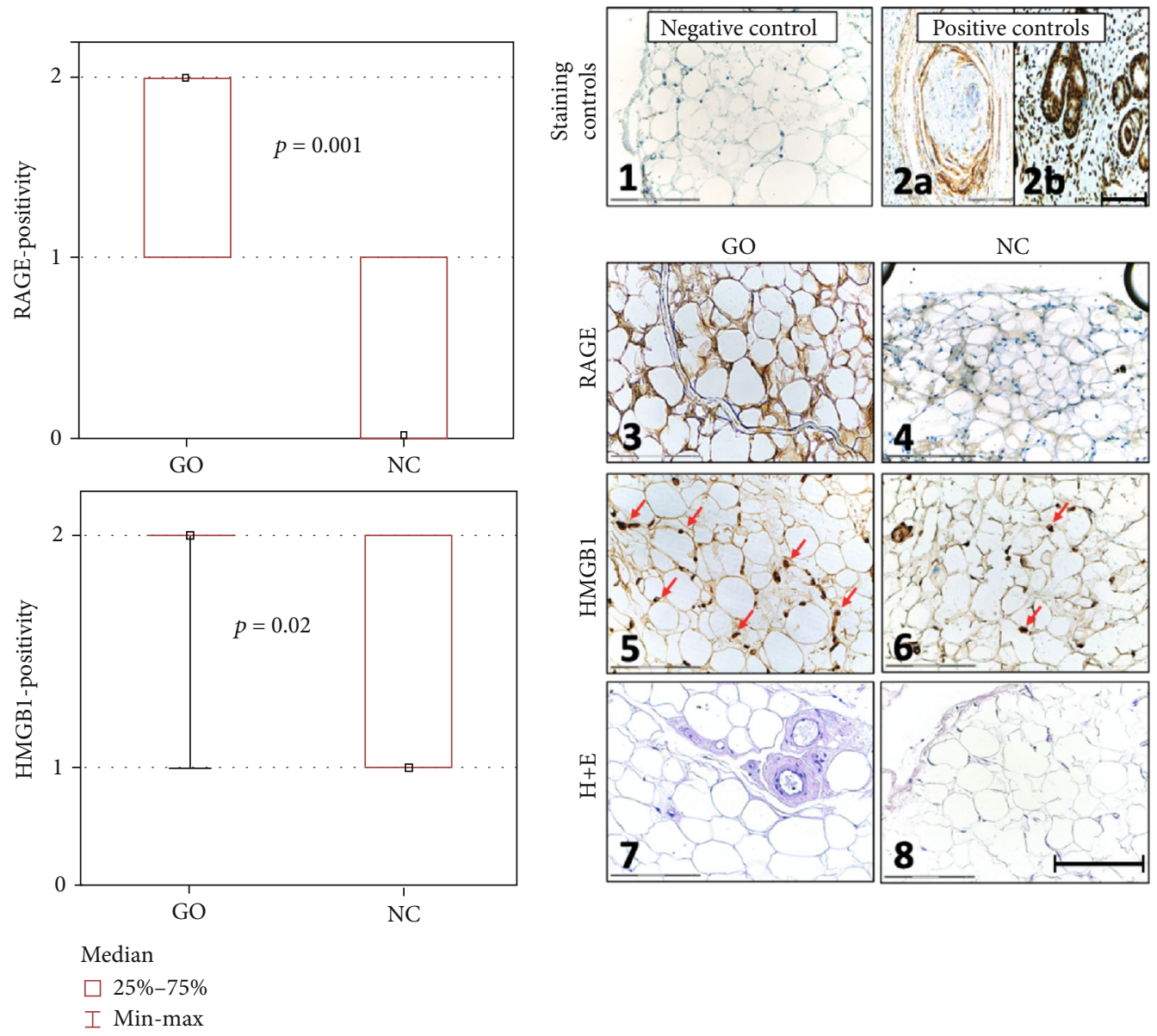

(a)

(b)

Figure 1: RAGE and HMGB1 expression in normal control (NC) and in Graves' ophthalmopathy (GO) tissues. (a) Statistical analysis of RAGE and HMGB1 expression in GO tissues compared to NC. The sections were scored as described in Material and Methods. (b) Representative images of immunohistochemistry staining. B1: isotype negative control of GO tissue. B2: positive control. (a) RAGE expression on head and neck squamous cell carcinoma tissue. (b) HMGB1 expression on nasal cavity mucosa. B3: RAGE expression in GO tissue. B4: RAGE expression in NC tissue. B5: HMGB1 expression in GO tissue (arrows). B6: HMGB1 expression in NC tissue (arrows). B7: hematoxylin and eosin $(\mathrm{H}+\mathrm{E})$ staining of GO tissue. B8: $\mathrm{H}+\mathrm{E}$ staining of $\mathrm{NC}$ tissue. $p$ value $\leq 0.05$ was considered to be significant. Bar $=150 \mu \mathrm{m}$.

in the activation of innate and adaptive immunity, development of inflammation, and increased production of cytokines. In patients with rheumatoid arthritis, the production of HMGB1 and the number of cells secreting HMGB1 at specific inflammation areas are elevated [19, 20]. Experimental models of arthritis showed inhibitory effects of antiHMGB1 antibodies on the development of synovial inflammation. HMGB1 is also found in SLE patients' plasma, and after being released from apoptotic cells, HMGB1 is bound to nucleosomes [21]. In turn, autoantibodies directed against nucleosomes and double-stranded DNA are characteristics for SLE. Our previous study demonstrated the expression of RAGE and HMGB1 in epithelial cells of sinonasal mucosa samples obtained from patients with chronic rhinosinusitis or in epithelial cells of middle ear cholesteatoma [12, 22]. We observed a strong correlation between the disease severity and RAGE expression.

Studies have shown that HMGB1 is involved in the pathogenesis of autoimmune thyroiditis and other chronic diseases by augmenting inflammation signaling and inflammatory infiltration [22-24]. Han et al. demonstrated that HMGB1 and its receptors are associated with the inflammatory mechanisms of GO and blocking of the HMGB1 pathway can be utilized to treat GO patients [25]. The authors demonstrated higher gene expression levels of RAGE and TLRs and higher mRNA and protein levels of HMGB1 in GO tissues compared to non-GO tissues. Moreover, blocking of HMGB1, RAGE, and TLR caused diminished production of proinflammatory cytokines confirming the participation of HMGB1 in GO inflammation. Plasma levels of HMGB1 were shown to correlate with the clinical activity score (CAS), indicating a valuable biomarker of disease activity. Being coherent with previous studies, we confirm the expression of RAGE and HMGB1 in GO tissues using immunohistochemistry. We observed a strong correlation between DON and RAGE expression in GO tissues. In addition, in GO tissues we demonstrated the presence of RAGE and HMGB1 positive inflammatory cells which were closely located to 


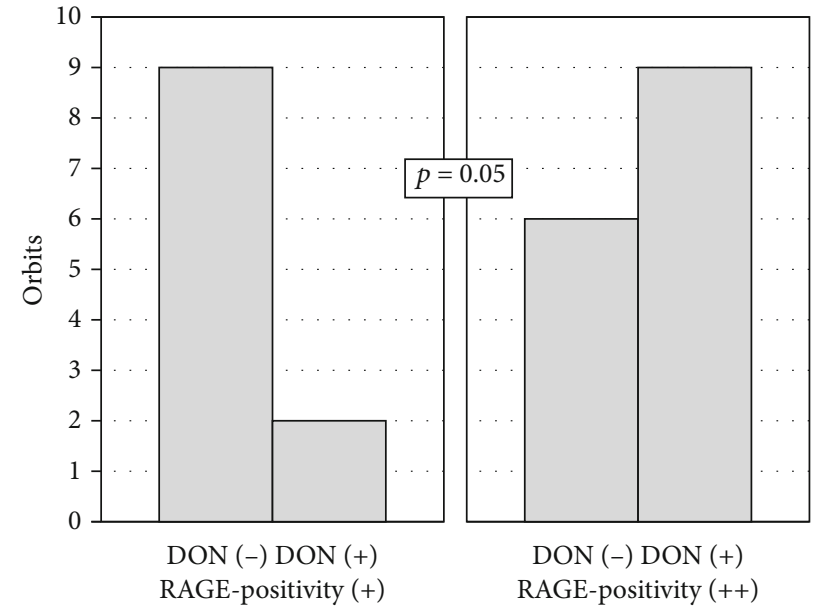

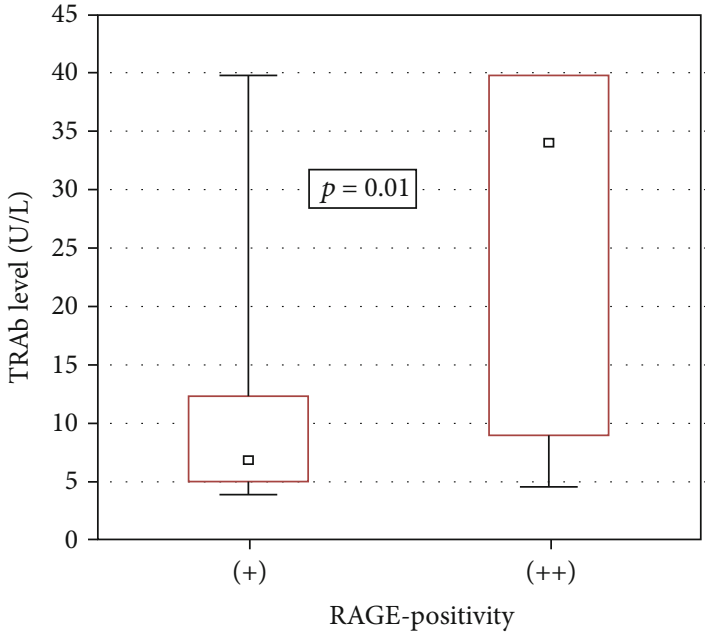

Median

$\square 25 \%-75 \%$

I Min-max

(a)

(b)

FIGURE 2: RAGE expression in correlation with clinical data. (a) RAGE positivity vs. occurrence of dysthyroid optic neuropathy (DON) $(p=0.05)$. (b) RAGE positivity vs. levels of TSHR antibodies (TRAb; $p=0.01$ ). $p$ value $\leq 0.05$ was considered to be significant.

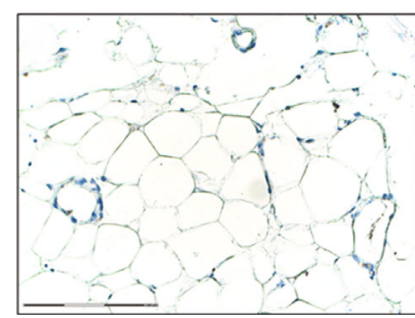

(a)

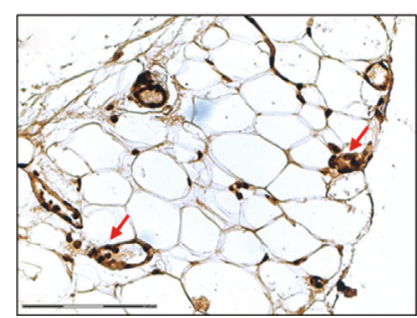

(c)

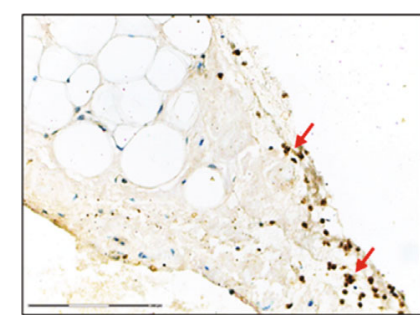

(b)

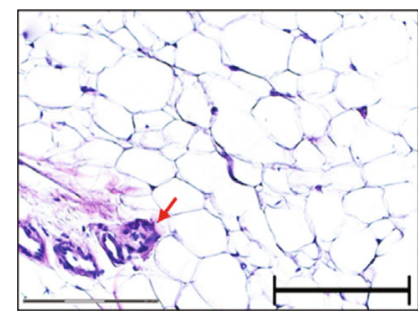

(d)

FIGURE 3: RAGE and HMGB1 expression on inflammatory infiltrates in GO tissues. (a) Isotype negative control staining of GO tissue. (b) RAGE expression on inflammatory infiltrates (arrows). (c) HMGB1 expression on inflammatory infiltrates (arrows). (d) H+E staining of GO tissue. Bar $=150 \mu \mathrm{m}$.

the vessels. We hypothesize that those cells may also play an important role in driving the inflammatory process. According to our knowledge, this is the first study that has shown the presence of RAGE and HMGB1 positive inflammatory cells in the GO adipose tissue microenvironment.

Peng et al. demonstrated that HMGB1 and RAGE expression on monocytes isolated from PBMCs was higher in patients with autoimmune thyroid diseases (AITD) compared to those isolated from healthy controls [26]. Their findings suggested a vital role of RAGE and HMGB1 in the pathogen- esis of AITD. RAGE participates in inflammatory cell recruitment and chronic inflammation development, which are considered crucial aspects of GO pathogenesis [25,27].

Pathogenesis of GO is closely associated with the presence of TRAb which involves autoantibodies directed against TSHR expressed not only on thyrocytes but also on orbital tissues. TRAb levels correlate with the clinical activity of GO [28]. Diana et al. reported a correlation between TRAb and the degree of ocular changes in GD [29]. However, the relationship between TRAb level and HMGB1 expression 
was not observed by us. In turn, Han et al. demonstrated a strong correlation between plasma level of HMGB1 and $\mathrm{TRAb}$ as well as CAS. Literature data report a correlation between RAGE levels in the serum and disease activity of other autoimmune diseases [30]. Studies analyzing the involvement of RAGE and HMGB1 in the pathogenesis of various inflammatory diseases are accompanied by experimental studies showing anti-RAGE/HMGB1 antibodies that prevent chronic inflammation $[31,32]$.

In summary, we demonstrated for the first time by using immunohistochemistry that RAGE and HMGB1 are overexpressed in GO patients in comparison with NC. We also observed a correlation between RAGE expression and occurrence of dysthyroid optic neuropathy (DON) or elevated levels of TSH Receptor Antibodies (TRAb) in GO patients. Overexpression of RAGE and HMGB1 might be associated with GO pathogenesis.

\section{Data Availability}

The research and clinical data used to support the findings of this study are included within the article.

\section{Conflicts of Interest}

The authors declare that they have no conflict of interest.

\section{Authors' Contributions}

M.J.S. designed the study; D.Ł. and A.G. conducted the experiments; M.J.S., I.K., K.B.P., K.C., P.M., A.J.P., and Z.S. collected the clinical data; M.M.G. statistically analyzed the data; M.J.S., N.L., P.M., A.J.P., and J.B. interpreted the data; D.E., M.J.S., and N.L. prepared the manuscript; M.J.S., N.L., D.Ł., and J.B. analyzed the literature; D.Ł. and M.J.S. funded the study.

\section{Acknowledgments}

The authors would like to express their thanks to Dr. Andrzej Kluk from the Department of Clinical Pathology, Poznan University of Medical Science, Poland, for his advice on the immunohistochemistry. The study was partially financed by the Medical University of Warsaw research grant $[\mathrm{MB} / \mathrm{M} / 39 /(66) \#]$ to D.E. and by the National Science Centre, Poland, research grant [UMO-2017/25/B/NZ5/02949\#] to M.J.S.

\section{References}

[1] T. J. Smith and L. Hegedus, "Graves' disease," The New England Journal of Medicine, vol. 375, no. 16, pp. 1552-1565, 2016.

[2] J. J. Khong, A. A. McNab, P. R. Ebeling, J. E. Craig, and D. Selva, "Pathogenesis of thyroid eye disease: review and update on molecular mechanisms," The British Journal of Ophthalmology, vol. 100, no. 1, pp. 142-150, 2016.

[3] D. Jain, S. Mor, H. K. Aggarwal, P. Chhabra, and P. Jain, "Thyroid association ophthalmopathy in Hashimoto's thyroiditis: a case report," Maedica (Bucur), vol. 12, no. 1, pp. 65-67, 2017.
[4] R. S. Bahn, "Graves' ophthalmopathy," The New England Journal of Medicine, vol. 362, no. 8, pp. 726-738, 2010.

[5] L. Bartalena, L. Baldeschi, K. Boboridis et al., "The 2016 European Thyroid Association/European Group on Graves' Orbitopathy Guidelines for the Management of Graves' Orbitopathy," European thyroid journal, vol. 5, no. 1, pp. 9-26, 2016.

[6] A. M. Schmidt, S. D. Yan, S. F. Yan, and D. M. Stern, "The multiligand receptor RAGE as a progression factor amplifying immune and inflammatory responses," The Journal of Clinical Investigation, vol. 108, no. 7, pp. 949-955, 2001.

[7] H. Rauvala and A. Rouhiainen, "RAGE as a receptor of HMGB1 (Amphoterin): roles in health and disease," Current Molecular Medicine, vol. 7, no. 8, pp. 725-734, 2007.

[8] G. P. Sims, D. C. Rowe, S. T. Rietdijk, R. Herbst, and A. J. Coyle, "HMGB1 and RAGE in inflammation and cancer," Annual Review of Immunology, vol. 28, no. 1, pp. 367-388, 2010.

[9] H. Yang, D. J. Antoine, U. Andersson, and K. J. Tracey, "The many faces of HMGB1: molecular structure-functional activity in inflammation, apoptosis, and chemotaxis," Journal of Leukocyte Biology, vol. 93, no. 6, pp. 865-873, 2013.

[10] U. G. Andersson and K. J. Tracey, "HMGB1, a proinflammatory cytokine of clinical interest: introduction," Journal of Internal Medicine, vol. 255, no. 3, pp. 318-319, 2004.

[11] R. Bahn, "The EUGOGO consensus statement on the management of Graves' orbitopathy: equally applicable to North American clinicians and patients," Thyroid, vol. 18, no. 3, pp. 281-282, 2008.

[12] K. Dzaman, M. Zagor, M. Molinska-Glura, and A. Krzeski, "High motility group box 1 (HMGB1) protein and its receptor for advanced glycation end products (RAGE) expression in chronic rhinosinusitis without nasal polyps," Folia Histochemica et Cytobiologica, vol. 53, no. 1, pp. 70-78, 2015.

[13] M. J. Szczepanski, M. Czystowska, M. Szajnik et al., "Triggering of Toll-like receptor 4 expressed on human head and neck squamous cell carcinoma promotes tumor development and protects the tumor from immune attack," Cancer Research, vol. 69, no. 7, pp. 3105-3113, 2009.

[14] D. Lacheta, P. Miskiewicz, A. Gluszko et al., "Immunological aspects of Graves' ophthalmopathy," BioMed Research International, vol. 2019, Article ID 7453260, 12 pages, 2019.

[15] Y. Hiromatsu, D. Yang, T. Bednarczuk, I. Miyake, K. Nonaka, and Y. Inoue, "Cytokine profiles in eye muscle tissue and orbital fat tissue from patients with thyroid-associated ophthalmopathy," The Journal of Clinical Endocrinology and Metabolism, vol. 85, no. 3, pp. 1194-1199, 2000.

[16] J. S. Yoon, M. K. Chae, S. Y. Lee, and E. J. Lee, “Anti-inflammatory effect of quercetin in a whole orbital tissue culture of Graves' orbitopathy," The British Journal of Ophthalmology, vol. 96, no. 8, pp. 1117-1121, 2012.

[17] D. Passali, E. Kern, R. Lei Chen, and L. Bellussi, "High mobility group box 1 (HMGB 1): a new protein in the pathogenesis of ENT inflammatory and infectious diseases," Acta Otorhinolaryngologica Italica, vol. 32, no. 1, pp. 46-47, 2012.

[18] D. Musumeci, G. N. Roviello, and D. Montesarchio, “An overview on HMGB1 inhibitors as potential therapeutic agents in HMGB1-related pathologies," Pharmacology \& Therapeutics, vol. 141, no. 3, pp. 347-357, 2014.

[19] R. Kokkola, J. Li, E. Sundberg et al., "Successful treatment of collagen-induced arthritis in mice and rats by targeting 
extracellular high mobility group box chromosomal protein 1 activity," Arthritis and Rheumatism, vol. 48, no. 7, pp. 20522058, 2003.

[20] R. Pullerits, I. M. Jonsson, G. Kollias, and A. Tarkowski, "Induction of arthritis by high mobility group box chromosomal protein 1 is independent of tumour necrosis factor signalling," Arthritis Research \& Therapy, vol. 10, no. 3, 2008.

[21] V. Urbonaviciute, B. G. Furnrohr, S. Meister et al., "Induction of inflammatory and immune responses by HMGB1nucleosome complexes: implications for the pathogenesis of SLE," The Journal of Experimental Medicine, vol. 205, no. 13, pp. 3007-3018, 2008.

[22] M. J. Szczepanski, M. Luczak, E. Olszewska et al., "Molecular signaling of the HMGB1/RAGE axis contributes to cholesteatoma pathogenesis," Journal of Molecular Medicine (Berlin, Germany), vol. 93, no. 3, pp. 305-314, 2015.

[23] C. Li, S. Peng, X. Liu et al., "Glycyrrhizin, a direct HMGB1 antagonist, ameliorates inflammatory infiltration in a model of autoimmune thyroiditis via inhibition of TLR2-HMGB1 signaling," Thyroid, vol. 27, no. 5, pp. 722-731, 2017.

[24] K. Dzaman, M. J. Szczepanski, M. Molinska-Glura, A. Krzeski, and M. Zagor, "Expression of the receptor for advanced glycation end products, a target for high mobility group box 1 protein, and its role in chronic recalcitrant rhinosinusitis with nasal polyps," Archivum Immunologiae et Therapiae Experimentalis (Warsz), vol. 63, no. 3, pp. 223-230, 2015.

[25] S. Y. Han, S. H. Choi, J. S. Shin, E. J. Lee, S. H. Han, and J. S. Yoon, "High-mobility group box 1 is associated with the inflammatory pathogenesis of Graves' orbitopathy," Thyroid, vol. 29, no. 6, pp. 868-878, 2019.

[26] S. Peng, C. Li, X. Wang et al., "Increased toll-like receptors activity and TLR ligands in patients with autoimmune thyroid diseases," Frontiers in Immunology, vol. 7, 2016.

[27] T. Chavakis, A. Bierhaus, N. Al-Fakhri et al., "The pattern recognition receptor (RAGE) is a counterreceptor for leukocyte integrins: a novel pathway for inflammatory cell recruitment," The Journal of Experimental Medicine, vol. 198, no. 10, pp. 1507-1515, 2003.

[28] M. N. Gerding, J. W. van der Meer, M. Broenink, O. Bakker, W. M. Wiersinga, and M. F. Prummel, "Association of thyrotrophin receptor antibodies with the clinical features of Graves' ophthalmopathy," Clinical Endocrinology, vol. 52, no. 3, pp. 267-271, 2000.

[29] T. Diana, R. S. Brown, A. Bossowski et al., "Clinical relevance of thyroid-stimulating autoantibodies in pediatric Graves' disease-a multicenter study," The Journal of Clinical Endocrinology and Metabolism, vol. 99, no. 5, pp. 1648-1655, 2014.

[30] M. R. Jafari Nakhjavani, M. Jafarpour, A. Ghorbanihaghjo, S. Abedi Azar, and A. Malek Mahdavi, "Relationship between serum-soluble receptor for advanced glycation end products (sRAGE) and disease activity in rheumatoid arthritis patients," Modern Rheumatology, vol. 29, no. 6, pp. 943-948, 2019.

[31] H. Schierbeck, P. Lundback, K. Palmblad et al., "Monoclonal anti-HMGB1 (high mobility group box chromosomal protein 1) antibody protection in two experimental arthritis models," Molecular Medicine, vol. 17, no. 9-10, pp. 1039-1044, 2011.

[32] H. Yang and K. J. Tracey, "Targeting HMGB1 in inflammation," Biochimica et Biophysica Acta, vol. 1799, no. 1-2, pp. 149-156, 2010. 